\title{
Combining Blood Glucose and SpO 2 /FiO 2 Ratio Facilitates Prediction of Imminent Ventilatory Needs in Emergency Room COVID-19 patients
}

\author{
Kazuya Sakai \\ Yokohama City University \\ Kai Okoda \\ Yokohama City University \\ Yokohama City University \\ Ryo Saji \\ Yokohama City University \\ Fumihiro Ogawa \\ Yokohama City University \\ Takeru Abe \\ Yokohama City University \\ Ichiro Takeuchi \\ Yokohama City University
}

Mototsugu Nishii ( $\sim$ s461211@yokohama-cu.ac.jp )

\section{Research Article}

Keywords: COVID-19, blood glucose, Japan

Posted Date: August 18th, 2021

DOI: https://doi.org/10.21203/rs.3.rs-801989/v1

License: (9) This work is licensed under a Creative Commons Attribution 4.0 International License.

Read Full License 


\section{Abstract}

The increasing requirement of mechanical ventilation (MV) due to the novel coronavirus disease (COVID19 ) is still a global threat. The aim of this study is to identify markers that can easily stratify the impending use of $\mathrm{MV}$ in the emergency room (ER). A total of 106 patients with COVID-19 requiring oxygen support were enrolled. Fifty-nine patients were provided MV 0.5 hours (interquartile range: 0.3 to 1.4 ) postadmission. Clinical and laboratory data before intubation were collected. Using a multivariate logistic regression model, we identified four markers associated with the impending use of $\mathrm{MV}$, including the ratio of peripheral blood oxygen saturation to fraction of inspired oxygen $\left(\mathrm{SpO}_{2} / \mathrm{FiO}_{2}\right.$ ratio), alanine aminotransferase, blood glucose (BG), and lymphocyte counts. Among these markers, the combination of $\mathrm{SpO}_{2} / \mathrm{FiO}_{2}$ ratio and $\mathrm{BG}$, which can be measured easily and immediately, showed higher accuracy in its prediction (AUC: 0.88 ) compared to $\mathrm{SpO}_{2} / \mathrm{FiO}_{2}$ ratio alone (AUC: 0.84 ). Moreover, even in patients without severe respiratory failure $\left(\mathrm{SpO}_{2} / \mathrm{FiO}_{2}\right.$ ratio $\left.>300\right), \mathrm{BG}(>138 \mathrm{mg} / \mathrm{dL})$ was predictive of $\mathrm{MV}$ use. Measuring $\mathrm{BG}$ and $\mathrm{SpO}_{2} / \mathrm{FiO}_{2}$ ratio may be a simple and versatile new strategy to accurately identify ER patients with COVID-19 at high risk for the imminent need of MV.

\section{Introduction}

At the end of 2019, an outbreak of a novel coronavirus (COVID-19) from Wuhan, China, quickly spread worldwide ${ }^{[1]}$. As a result, the World Health Organization (WHO) declared COVID-19 a pandemic on March 11,2020 . It has caused numerous infections and deaths, mainly in Europe and the United States. As of July 1,2021 , more than 180 million cases have been reported worldwide, with more than 4 million deaths [2]. Although the pathogenesis of COVID-19 has been elucidated and vaccines have been developed worldwide, it is yet to be eradicated.

COVID-19 has impacted healthcare delivery models worldwide. Many countries have experienced considerable difficulties with unexpected increases in cases, and several hospitals have reached or exceeded their capacities ${ }^{[3]}$. Approximately $30 \%$ of symptomatic patients unexpectedly progress to severe respiratory failure requiring hospitalization ${ }^{[4]}$. Therefore, medical resources such as intensive care unit (ICU) beds and ventilators are indispensable, but not inexhaustible. So far, many studies have developed a model to predict mortality and the need for the ventilatory management of patients with mild

to moderate COVID-19 ${ }^{[5-14]}$. However, no attempt has been made to easily and immediately predict in the emergency room (ER) whether an individual patient with severe COVID-19 requiring oxygen support is at imminent risk of progressing to critically ill COVID-19 requiring intubation and mechanical ventilation (MV).

In the present study, we hypothesized that few markers that can be immediately and easily measured would be helpful for prompt triage of patients with COVID-19 in the ER, and our data showed clinical utility of measuring blood glucose (BG) level and the ratio of peripheral blood oxygen saturation to 
fraction of inspired oxygen $\left(\mathrm{SpO}_{2} / \mathrm{FiO}_{2}\right.$ ratio: $\mathrm{S} / \mathrm{F}$ ratio) in risk stratification of imminent need for intubation or MV.

\section{Results}

\section{Baseline characteristics}

From February 2020 to July 2021, 107 patients with severe COVID-19 pneumonia were enrolled. Of these, 1 patient were excluded analysis because of missing data. Ultimately, a total of 106 patients were evaluated in this study (Fig. 1). Table 1 shows individual baseline clinical and outcome data in the present study population. The median age was 66 years (interquartile range [IQR]: 55 to 72). Of these patients, 77 (73\%) were male and 29 (27\%) were female. Hemodynamics indicated by blood pressure (BP) and heart rate (HR) was preserved at hospital arrival. More than $80 \%$ of the study population was diagnosed as pneumonia. Comorbidity was shown, including interstitial pneumonia, chronic obstructive pulmonary disease, hypertension, diabetes, chronic kidney disease, heart failure, and liver cirrhosis. More than $50 \%(n=59 / 106)$ required MV for severe respiratory failure 0.5 hours (IQR: 0.3 to 1.4$)$ after hospital admission (vented group), while the remaining patients did not during hospitalization (unvented group).

\section{Comparisons of clinical and laboratory data}

There were not any significant differences of age, sex, body mass index (BMI), and smoking between vented group and unvented group. With regarding to hemodynamics at hospital arrival, BP and HR did not show any significant differences between the 2 groups. However, respiratory status, as indicated by S/F ratio, was significantly worse before intubation in the vented group compared with in the unvented group. Alternatively, there were no significant differences in the prevalence of comorbidities between the two groups. Comparisons of laboratory data between the 2 groups showed significant increases in aspartate aminotransferase (AST), alanine aminotransferase (ALT), alkaline phosphatase (ALP), lactate dehydrogenase (LDH), blood urea nitrogen (BUN), C-reactive protein (CRP), and BG and decreases in the lymphocyte counts in the vented group (Table 2).

\section{Prediction for the impending MV use}

We evaluated the associations of significant nine variables in the univariate analysis, including $S / F$ ratio, lymphocyte count, AST, ALT, ALP, LDH, BUN, BG, and CRP with the use of MV using the area under receiver operating characteristic curve (AUROC) obtained from a 10-split crossover test. The combination of these nine variables showed an AUC of 0.89 [0.75-1.00] with a sensitivity of 0.91 , specificity of 0.81 , positive predictive value (PPV) of 0.90 , and negative predictive value (NPV) of 0.83 (Table 3 ).

The beta regression coefficient selected from a multivariable logistic regression model using these variables is shown in the supplemental Fig. 1. Based on significant beta regression coefficient with $>0.5$ 
of their values, we identified the top four variables to predict MV use, including S/F ratio, BG, lymphocyte counts, and ALT. The combination of the four variables showed higher accuracy (AUC of 0.89 [0.83-0.95]) compared to S/F ratio or BG alone (AUC of 0.84 [0.76-0.91] or AUC of 0.75 [0.66-0.91], respectively) (Fig. 2 , Table 4), with a sensitivity of 1.00 , specificity of 0.82 , PPV of 0.85 , and NPV of 1.00 (Table 4).

We further evaluated a predictive value of combing $S / F$ ratio and $B G$, which can be easily and immediately measured, on the use of MV. The accuracy of this combination in the prediction (AUC: 0.88 [0.82-0.94) was nearly equal to that of the combing 4 variables, with a sensitivity of 1.00 , specificity of 0.73 , PPV of 0.79 , and NPV of 1.00 (Table 4). A novel combination of S/F ratio and BG is likely to allow accurately and easily predict the impending MV use in not only ER but also ambulance and home.

Clinical utility of combining BG level and S/F ratio at hospital admission was tested with Kaplan-Meier event-incidence curves of MV use that were constructed according to above or below optimal cutoffs defined by AUROC analysis (BG: $138 \mathrm{mg} / \mathrm{dL}, \mathrm{S} / \mathrm{F}$ ratio: 300 ). In high-risk patients with low S/F ratio ( $\leqq$ 300), MV use rate at the 3-day follow-up period did not show a significant difference between low BG (< $138 \mathrm{mg} / \mathrm{dL})$ and high $B G(\geqq 138 \mathrm{mg} / \mathrm{dL})(\mathrm{n}=11 / 14(79 \%)$ vs. $\mathrm{n}=34 / 37(92 \%)$, respectively, $\mathrm{P}=0.48)$. However, in patients with high S/F ratio $(>300)$, those with high $B G$ had a significantly higher MV use rate compared to those with low BG ( $n=10 / 20(50 \%)$ vs. $n=4 / 35$ (11\%), respectively, $P<0.01)$ (Fig. 3). Importantly, this suggests that measuring BG level at hospital admission allows identifying patients at high risk for impending MV use from population with preserved respiratory status, which supports a better accuracy of combining $B G$ and $S / F$ ratio than $S / F$ ratio alone.

\section{Discussion}

Our study focused on the possibility of easily identifying ER patients with COVID-19 at high risk for the impending use of ventilation using easily and immediately measurable biomarkers at the time of admission. Our data showed for the first time that measuring BG levels and S/F ratio may facilitate the stratification of high-risk patients in the ER.

It has been demonstrated that hyperglycemia in patients with COVID-19 is associated with a higher risk of $M V$ use or in-hospital mortality ${ }^{[15-19]}$. However, its predictive role in impending use of MV remained elusive. Presence of severe respiratory failure, as indicated by low S/F ratio, is strongly predictive of impending use of MV. Our data supported its robust predictive value. On the other hand, in our study, $25 \%$ $(n=14 / 55)$ of patients who preserved respiratory status under oxygen support ( $F / S$ ratio $>300)$ at hospital arrival imminently required the MV use 0.5 hours post-admission, indicating the limitation of $S / F$ ratio as a predictor in COVID-19. Importantly, our data showed that measuring BG level in the ER may allow easily identifying such high-risk patients, prior to the development of severe respiratory failure. Our study revealed for the first time the potential of BG level, which can be easily and quickly measured in the $E R$, as a predictor of imminent MV use. 
It has been reported that hyperglycemia in COVID-19 patients is associated with a higher risk of inhospital mortality regardless prior history or presence of diabetes ${ }^{[19]}$. In infectious diseases, secretion of catecholamines from the adrenal medulla leads to hyperglycemia, indicating increased systemic stress [20]. In turn, the increased glucose metabolism imposed by sustained hyperglycemia seems to enhance SARS-CoV-2's entry and subsequent replication, as well as exacerbated immune responses such as tumor necrosis factor- $\alpha$, interleukin (IL)-1 $\beta$, and IL-6. ${ }^{21-22}$ Thus, a disrupted glucose metabolism and metabolic derangement may be an intrinsic cellular strategy that favors SARS-CoV-2 pathogenesis. ${ }^{[22]}$ Therefore, hyperglycemia may not only be a result of in vivo reactions, but it may also be involved in the enhancement of immune responses and excessive immune responses, such as those seen in COVID-19.

Our study exhibited several strengths. First, our results were comparable to those of previously reported predictive models. Second, we developed a simplified but highly predictive model to improve the clinical utility of this model and the required factors are easy to obtain and can be implemented immediately. Blood glucose can be measured with a meter developed for self-monitoring of blood glucose

(SMBG) in diabetic patients, and the S/F ratio can be easily calculated from a pulse oximeter. By using these simple measurement devices, these variables are possible to measure outside the hospital. Therefore, in a pandemic setting where adequate medical care cannot be supplied, this model can be used to screen for severe disease in places such as homes and clinics without adequate medical equipment.

This study has several limitations. Because our study dataset was a backward-looking study, only the parameters listed in the electronic medical records could be analyzed, and there were several missing values. In addition, because it was a single-center study, we could not evaluate the generalizability of the machine learning model. Further research is needed to determine the applicability of the newly developed machine learning models in clinical practice. In addition, the race of the patients was biased toward the Japanese. Because previous literature has reported that there were racial differences in the severity of COVID-19 cases, it is necessary to verify whether the developed model can be applied in countries other than Japan. The blood glucose value used in this study was measured at the hospital, not the blood glucose value measured by the meter for SMBG. Past studies have shown that capillaries correlate with plasma glucose levels, and the results of this study may be applicable to simple blood glucose meters [23]

\section{Methods}

\section{Study design}

This was a retrospective observational study conducted at one hospital in Japan. Patients with severe COVID-19 pneumonia requiring oxygen support who were admitted to the Yokohama City University Hospital (YCUH) between February 2020 and July 2021 were enrolled in this study. COVID-19 pneumonia was diagnosed by via Polymerase Chain Reaction (PCR) and chest Xp. Enrolled patients were observed 
during 30-day after the enrollment to evaluate clinical outcome. The primary outcome was the use of MV. Patients with missing data, those who offered to withdraw from the study, pregnant women, age $<20$ years, and those who did not require oxygen support in the ER were excluded from the evaluation. With complete clinical, laboratory, and outcome data and oxygen support in the ER as well as consent for participation, a total of 106 COVID-19 patients were included in the final analysis.

\section{Ethical considerations}

This study was approved by the Institutional Ethics Board of the Yokohama City University Hospital (No. B210100010). All research was performed in accordance with the relevant guidelines and regulations. During hospitalization, patients were provided negative and positive information regarding this study, including the purpose and contribution of this study, the use of personal information, and complications associated with blood collection, and were asked to participate in this study. Ultimately, we obtained written informed consent for participation in the study and access to medical and laboratory records from patients. The study had no risk/negative consequence on those who participated in the study. Medical record numbers were used for data collection and no personal identifiers were collected or used in the research report. Data was accessed from February 16,2020 , to July 5, 2021, and access to the collected information was limited to the principal investigator and confidentiality was maintained throughout the project.

\section{Data and Specimen collection}

We obtained clinical and laboratory in the ER, and treatment and outcome data were obtained from electronic medical records. Two researchers independently reviewed the data collection forms to doublecheck the collected data.

\section{Definition of severity by COVID-19}

The illness severity of COVID-19 was defined according to the Ministry of Health, Labor, and Welfare in Japan, which defines critically ill patients as those requiring ventilators or treatment in intensive care units ${ }^{[24]}$. The need for $\mathrm{MV}$ was determined by the ratio of the partial pressure of arterial oxygen to $\mathrm{FiO}_{2}$ $\left(\mathrm{PaO}_{2} / \mathrm{FiO}_{2}\right)$ and respiratory pattern; a MV was introduced if $\mathrm{PaO}_{2} / \mathrm{FiO}_{2}$ was less than 200 or if $\mathrm{PaO}_{2} / \mathrm{FiO}_{2}$ was less than 250 and excessive effort breathing continued.

\section{Development of the model}

The following methods were used to develop the predictive model: (1) data preprocessing and (2) variable selection and model evaluation. 


\section{Data preprocessing}

Covariates missing more than $5 \%$ of their data were excluded. In the case of highly correlated pairs of variables (correlation coefficient $>0.8$ ), a variable with a higher missing rate were removed. For missing values, median values were imputed.

\section{Variable selection and model evaluation}

The data set was divided into vented and non-vented groups. Univariate analysis was performed for each of variable to extract risk factors for predicting severe disease. In addition, the covariates reported in previous studies were used as independent variables. To identify significant variables associated with the severity, a multivariable logistic regression model was used. The beta regression coefficient selected from this model is shown in the supplemental Fig. 1. We selected common components of these models, based on significant beta regression coefficient with $>0.5$ of their values. Next, we developed a simplified prognostic model using an interaction from two variables ( $S / F$ ratio, $B G$ ), because they were easy to use in clinical practice. We evaluated the performances of the models using the area under the receiver operating curve, AUROC, sensitivity, and specificity values obtained via 10-fold cross-validation. The sensitivity, specificity, or AUC performances were defined as poor with a value $<0.5$, low with a value between 0.5 and 0.7 , moderate with a value between 0.7 and 0.85 , and excellent with a value $>0.85$.

We used Python (3.7.10) for data collection, data cleaning, functional engineering, and machine-learning training and testing. The development environment included JupyterNotebook. The main libraries included Numpy, Pandas, Sklearn, Scipyand matplotlib. To prevent overfitting, we used k-split crosstesting and hyperparameter optimization during training.

\section{Statistical analysis}

Data analysis was performed using the Python software version 3.7.10. Dependent variables were presented as the median (IQR) for continuous variables frequencies (\%) for categorical variables. Differences between the vented and non-vented groups were analyzed with Fisher's exact test for categorical data or the Mann-Whitney U test for continuous data. Receiver operating curve, ROC, analysis and the AUROC were used to evaluate the ability of each scoring system to predict an increase in ventilation. To test an interaction between S/F ratio and BG on event-free survival, Kaplan Meier survival curve was drawn, and log-rank tests with multiple comparisons were performed. We determined $p<0.05$ as statistically significant.

\section{Declarations}

\section{Acknowledgments}


This research was supported by AMED under Grant Number 20fk0108405h0001.

\section{Authors' contributions}

$\mathrm{KS}$ and KO prepared the manuscript, collected the references, and performed the ML analysis. MN organized and reviewed the manuscript. FO coordinated all authors. TA provided epidemiological insight into the study and performed statistical analysis for this study. RS provided clinical support.

\section{Corresponding author}

Correspondence to Mototsugu Nishii

\section{Competing interests:}

there are no conflicts of interest regarding the publication of this paper.

\section{References}

1. Wu, J. T., Leung, K. \& Leung, G. M. Nowcasting and forecasting the potential domestic and international spread of the 2019-nCoV outbreak originating in Wuhan, China: A modelling study. Lancet, 395, 689-697 https://doi.org/10.1016/S0140-6736(20)30260-9 (2020).

2. COVID-19 dashbord by the Center for Systems Science and Engineering (CSSE) at johns Hopkins universtity. https://gisanddata.maps.arcgis.com/apps/dashboards/bda7594740fd40299423467b48e9ecf6.

3. Daily, C. \& Report https://www.who.int/emergencies/diseases/novel-coronavirus-2019/situationreports/.

4. Huang, C. et al. Clinical features of patients infected with 2019 novel coronavirus in Wuhan, China. Lancet 395, 497-506(2020). 10.1016/S0140-6736(20)30183-5, Pubmed:31986264.

5. Mehraeen, E. et al. Predictors of mortality in patients with COVID-19-a systematic review. Eur. J. Integr. Med. 40, 101226(2020). 10.1016/j.eujim.2020.101226, Pubmed:33101547.

6. Jimenez-Solem, E. et al. Developing and validating COVID-19 adverse outcome risk prediction models from a bi-national European cohort of 5594 patients. Sci. Rep. 11, 3246(2021). 10.1038/s41598-021-81844-x, Pubmed:33547335.

7. Zoabi, Y., Deri-Rozov, S. \& Shomron, N. Machine learning-based prediction of COVID-19 diagnosis based on symptoms. npj Digit. Med, 4, 3 https://doi.org/10.1038/s41746-020-00372-6 (2021).

8. Alballa, N. \& Al-Turaiki, I. Machine learning approaches in COVID-19 diagnosis, mortality, and severity risk prediction: A review. Inform. Med. Unlocked, 24, https://doi.org/10.1016/j.imu.2021.100564 (2021). 
9. Drosten, C. et al. Identification of a novel coronavirus in patients with severe acute respiratory syndrome. N. Engl. J. Med. 348, 1967-1976(2003). 10.1056/NEJMoa030747, Pubmed:12690091.

10. Zaki, A. M., van Boheemen, S., Bestebroer, T. M., Osterhaus, A. D. \& Fouchier, R. A. Isolation of a novel coronavirus from a man with pneumonia in Saudi Arabia. N. Engl. J. Med. 367, 1814-1820(2012). 10.1056/NEJMoa1211721, Pubmed:23075143.

11. Wang, D. et al. Clinical characteristics of 138 hospitalized patients with 2019 novel coronavirusinfected pneumonia in Wuhan, China. JAMA. 323, 1061-1069(2020). 10.1001/jama.2020.1585, Pubmed:32031570.

12. Huang, C. et al. Clinical features of patients infected with 2019 novel coronavirus in Wuhan, China. Lancet, 395 (20), )30183-5, Pubmed:31986264.497-506 https://doi.org/10.1016/S0140-6736 (2020).

13. Booth, A. L., Abels, E. \& McCaffrey, P. Development of a prognostic model for mortality in covid-19 infection using machine learning. Mod. Pathol. 34, 522-531(2021). 10.1038/s41379-020-00700-x, Pubmed:33067522.

14. Bertsimas, D. et al. Covid-19 mortality risk assessment: An international multi-center study. PLOS ONE. 15, e0243262(2020). 10.1371/journal.pone.0243262, Pubmed:33296405.

15. Rechtman, E., Curtin, P., Navarro, E., Nirenberg, S. \& Horton, M. K. Vital signs assessed in initial clinical encounters predict covid-19 mortality in a nyc hospital system. Sci. Rep. 10, 21545(2020). 10.1038/s41598-020-78392-1, Pubmed:33298991.

16. Sze, S. et al. Ethnicity and Clinical Outcomes in COVID-19: A Systematic Review and Meta-Analysis. Eclinical. Medicine(2020). doi: 10.1016/j.eclinm.2020.100630.

17. Hoyt et al. Prediction of respiratory decompensation in Covid-19 patients using machine learning: The READY trial. Comput. Bio. Med. 124, 103949(2020). 10.1016/j.compbiomed.2020.103949, Pubmed: 32798922.

18. Wen et al. Early predictors for mechanical ventilation in COVID-19 patients. Ther. Adv. Respir. Dis. 14, 1753466620963017(2020). 10.1177/1753466620963017, Pubmed: 33054630.

19. Huang, I., Lim, M. A. \& Pranata, R. Diabetes mellitus is associated with increased mortality and severity of disease in COVID-19 pneumonia - A systematic review, meta-analysis, and metaregression. Diabetes. Metab. Syndr, 14, 395-403 https://doi.org/10.1016/j.dsx.2020.04.018 (2020).

20. Nandy, K. et al. Coronavirus disease (COVID-19): A systematic review and meta-analysis to evaluate the impact of various comorbidities on serious events. Diabetes. Metab. Syndr, 14, 1017-1025 https://doi.org/10.1016/j.dsx.2020.06.064 (2020).

21. Norouzi, M. et al. Type-2 diabetes as a risk factor for severe COVID-19 infection. Microorganisms. 9(2021). 10.3390/microorganisms9061211, Pubmed:34205044.

22. Mamtani, M. et al. Association of hyperglycaemia with hospital mortality in nondiabetic COVID-19 patients: A cohort study. Diabetes. Metab. 47, 101254(2021). 10.1016/j.diabet.2021.101254, Pubmed:33781926. 
23. Carrasco-Sánchez, F. J. et al. Admission hyperglycaemia as a predictor of mortality in patients hospitalized with COVID-19 regardless of diabetes status: data from the Spanish SEMI-COVID-19 Registry. Ann. Med, 53, 103-116 https://doi.org/10.1080/07853890.2020.1836566 (2021).

24. Weil, M. H. \& Shubin, H. Treatment of shock caused by bacterial infections. Calif. Med, 119, 7-13 (1973). Pubmed:4755702.

25. Codo, A. C. et al. Elevated glucose levels favor SARS-CoV-2 infection and monocyte response through a HIF-1alpha/glycolysis-dependent axis. Cell Metab, 32, 437-446 (2020).

26. Ardestani, A. \& Azizi, Z. Targeting glucose metabolism for treatment of COVID-19. Signal. Transduct. Target. Ther, 6, 112 https://doi.org/10.1038/s41392-021-00532-4 (2021).

27. Kuwa, K., Nakayama, T., Hoshino, T. \& Tominaga, M. Relationships of glucose concentrations in capillary whole blood, venous whole blood and venous plasma. Clin. Shim. Acta, 307, 187-192 https://doi.org/10.1016/s0009-8981 (2001).

28. Clinical management of patients with COVID-19. https://www.mhlw.go.jp/content/000646531.pdf.

\section{Tables}


Table 1

\section{Clinical Characteristics}

\section{Variables}

Age (years)

Gender

BMl

Smoking

\section{Vital sign at hospital arrival}

SBP $(\mathrm{mmHg})$

DBP $(\mathrm{mmHg})$

BT $\left({ }^{\circ} \mathrm{C}\right)$

HR (beat/min)

$\mathrm{RR}(/ \min )$

$\mathrm{SpO}_{2}$ to $\mathrm{FiO}_{2}$ ratio
Total patients $(n=106)$

66 [55-72]

male:77 (72.6)

female:29 (27.4)

$24.0[225-27.0]$

29 (27.4)
137 [122-156]

81 [71-88]

37 [37-38]

90 [78-104]

24 [20-28]

$308[117-457]$

\section{Past medical history}

IP

COPD

Asthma

HT

DM

CKD

Heart disease

$\mathrm{HF}$

dialysis

LC
$1(0.9)$

$3(2.8)$

13 (12.3)

43 (40.6)

32 (30.2)

19 (17.9)

$9(8.5)$

3 (2.8)

16 (15.1)

7 (6.6) 


\section{Outcome data}

Mechanical ventilation

$59(56)$

Hours until intubation after hospital admission (hours)

$0.5[0.3-1.4]$

All categorical variables were presented as $\mathrm{n}(\%)$. Continuous variables are shown as median values and [interquartile ranges]. BMI, body mass index; SBP, systolic blood pressure; DBP, diastolic blood pressure; $\mathrm{BT}$, body temperature; $\mathrm{HR}$, heart rate; $\mathrm{RR}$, respiratory rate; $\mathrm{SpO}_{2 \bigotimes \text {, }}$, peripheral oxygen saturation; $\mathrm{IP}$, interstitial pneumonia; COPD, chronic obstructive pulmonary disease; HT, hypertension; DM, diabetes; CKD, chronic kidney disease; HF, heart failure; LC, liver cirrhosis. 
Table 2

Comparisons of clinical and laboratory data

\begin{tabular}{|c|c|c|c|}
\hline Variables & Unvented $(n=47)$ & Vented $(n=59)$ & $P$ value \\
\hline Age (years) & 66 [49-73] & $65[57-72]$ & 0.98 \\
\hline \multirow[t]{2}{*}{ Gender } & male:31 (66.0) & male:46 (78.0) & \multirow[t]{2}{*}{0.17} \\
\hline & female:16 (34.0) & female:13 (22.0) & \\
\hline BMI & $23.9[21.8-26.8]$ & $24.3[22.7-27.8]$ & 0.63 \\
\hline Smoking & $11(23.4)$ & $18(30.5)$ & 0.41 \\
\hline \multicolumn{4}{|c|}{ Vital sign at hospital arrival } \\
\hline $\mathrm{SBP}(\mathrm{mmHg})$ & 138 [123-155] & 136 [120-157] & 0.75 \\
\hline $\mathrm{DBP}(\mathrm{mmHg})$ & $81[70-88]$ & 80 [72-88] & 0.87 \\
\hline BT $\left({ }^{\circ} \mathrm{C}\right)$ & $37.7[37.1-38.2]$ & $37.2[36.7-37.8]$ & 0.05 \\
\hline HR (beat/min) & $91.5[82.2-106.0]$ & $85[73-100]$ & 0.07 \\
\hline $\mathrm{RR}(/ \min )$ & 24 [20-25] & 25 [20-30] & 0.93 \\
\hline $\mathrm{SpO}_{2}$ to $\mathrm{FiO}_{2}$ ratio & 452 [388-462] & 155 [95-284] & $<0.01$ \\
\hline
\end{tabular}

\section{Past medical history}

\begin{tabular}{llll}
\hline IP & $1(2.1)$ & $0(0)$ & 0.26 \\
\hline COPD & $2(4.3)$ & $1(1.7)$ & 0.43 \\
\hline Asthma & $4(8.5)$ & $9(15.3)$ & 0.29 \\
\hline HT & $15(31.9)$ & $28(47.5)$ & 0.11 \\
\hline DM & $12(25.5)$ & $20(33.9)$ & 0.35 \\
\hline CKD & $9(19.1)$ & $10(16.9)$ & 0.77 \\
\hline Heart disease & $6(12.8)$ & $3(5.1)$ & 0.16 \\
\hline HF & $1(2.1)$ & $2(3.4)$ & 0.67 \\
\hline dialysis & $9(19.1)$ & $7(11.9)$ & 0.30 \\
\hline LC & $3(6.4)$ & $4(6.8)$ & 0.93 \\
\hline
\end{tabular}




\section{Laboratory data}

Lymphocyte $(/ \mu \mathrm{L})$

Red Blood Cell

$\left(\times 10^{\wedge} 6 / \mu \mathrm{L}\right)$

Platelet $\left(\times 10^{\wedge} 3 / \mu \mathrm{L}\right)$

Hemoglobin $(\mathrm{g} / \mathrm{dL})$

PT-INR

APTT (sec)

FDP-D dimer

$(\mu \mathrm{g} / \mathrm{mL})$

Total bilirubin

(mg/dL)

AST (U/L)

ALT (U/L)

$\operatorname{ALP}(\mathrm{U} / \mathrm{L})$

$\mathrm{LDH}(\mathrm{U} / \mathrm{L})$

CK (U/L)

BUN (mg/dL)

Creatinine $(\mathrm{mg} / \mathrm{dL})$

e-GFR

$(\mathrm{mL} / \mathrm{min} / 1.73)$

$\mathrm{Na}(\mathrm{mmol} / \mathrm{L})$
$\mathrm{K}(\mathrm{mmol} / \mathrm{L})$
$\mathrm{Cl}(\mathrm{mmol} / \mathrm{L})$
Glucose $(\mathrm{mg} / \mathrm{dL})$
$\mathrm{CRP}(\mathrm{mg} / \mathrm{dL})$

Procalcitonin $(\mathrm{ng} / \mathrm{mL})$

Troponin I $(\mathrm{pg} / \mathrm{mL})$

CK-MB (U/L)

HbA1c (\%)

All categorical variables were presented as $\mathrm{n}(\%)$. Continuous variables are shown as median values
$785.5[591.2-1087.7] \quad 535.0[304.1-777.5] \quad<0.01$

$4.34[3.98-4.78] \quad 4.31[3.93-4.73] \quad 0.70$

174.0 [125. 5-209.0]

$191.0[154.0-253.5]$

0.14

$13.6[12.2-15.0]$

$13.4[12.1-14.4]$

0.31

$1.11[1.04-1.24]$

1.21 [1.10-1.28]

0.05

34.0 [30.8-36.0]

34.0 [31.2-37.4]

0.50

0.91 [0.50-3.05]

1.24 [0.86-2.62]

0.96

$0.6[0.4-0.9]$

$0.5[0.4-0.8]$

0.98

31.0 [21.5-42.0]

41.0 [32.5-69.0]

$<0.01$

19.0 [15.0-29.5]

41.0 [19.0-63.5]

$<0.01$

104 [75-195]

83 [59-125]

0.04

261.0 [208.5-346.5]

87.0 [61.5-204]

434.0 [355.5-517.0]

$<0.01$

106.0 [56.0-246.5]

0.83

16 [13-30]

22 [17-31]

0.04

0.91 [0.71-1.88]

0.78 [0.63-1.16]

0.06

67.0 [28.9-85.2]

78.7 [46.2-98.0]

0.08

139 [136-141]

139 [136-142]

0.38

4.0 [3.6-4.2]

0.26

102 [100-104]

103 [99-105]

0.16

118 [106-146]

160 [138-219]

$<0.01$

$3.7[0.8-6.8]$

9.4 [3.6-14.17]

$<0.01$

0.08 [0.05-0.47]

0.16 [0.07-0.33]

0.19

11.4 [4.7-73.2]

6.4 [4.7-21.5]

0.10

5 [5-5]

$5[5-5]$

0.47

6.0 [5.7-6.4]

6.3 [6.0-7.5]

0.67
4.0 [3.7-4.3] 
and [interquartile ranges]. BMI, body mass index; SBP, systolic blood pressure; DBP, diastolic blood pressure; $\mathrm{BT}$, body temperature; $\mathrm{HR}$, heart rate; $\mathrm{RR}$, respiratory rate; $\mathrm{SpO}_{2 \bigotimes \text {, }}$ peripheral oxygen saturation; $I P$, interstitial pneumonia; COPD, chronic obstructive pulmonary disease; $\mathrm{HT}$, hypertension; DM, diabetes; CKD, chronic kidney disease; HF, heart failure; LC, liver cirrhosis; PT-INR, prothrombin timeinternational normalized ratio; APTT, activated partial thromboplastin time; AST, aspartate aminotransferase; ALT, alanine aminotransferase; ALP, alkaline phosphatase; LDH, lactate dehydrogenase; $\mathrm{CK}$, creatine kinase; $\mathrm{BUN}$, blood urea nitrogen; CRP, C-reactive protein; CK-MB, creatine kinase MB.

\section{Table 3}

Receiver operating characteristic analyses for association of combined nine variables with mechanical ventilation use.

\begin{tabular}{|c|c|c|c|c|c|}
\hline Variables & AUC & Sensitivity & Specificity & NPV & PPV \\
\hline $\begin{array}{l}\text { S/F, Lym, AST, ALT, ALP, LDH, BUN, BG, } \\
\text { and CRP }\end{array}$ & $\begin{array}{l}0.89[0.75- \\
1.00]\end{array}$ & 0 & 0.8 & 0.83 & 0 \\
\hline
\end{tabular}

AUC, area under the curve; NPV, Positive Negative Value; PPV, Positive Predictive Value; S/F, $\mathrm{SpO}_{2}$ $/ \mathrm{FiO}_{2}$ ratio; Lym, lymphocyte counts; $\mathrm{AST}$, aspartate aminotransferase; ALT, alanine aminotransferase; ALP, alkaline phosphatase; LDH, lactate dehydrogenase; BUN, blood urea nitrogen; BG, blood glucose; CRP, C-reactive protein.

Table 4

Receiver operating characteristic analyses for association of different four variables or combined variables with mechanical ventilation use.

\begin{tabular}{llllll}
\hline Variables & AUC & Sensitivity & Specificity & NPV & PPV \\
\hline S/F, BG, Lym, and ALT & $0.89[0.83-0.95]$ & 1.00 & 0.82 & 1.00 & 0.85 \\
\hline S/F, BG & $0.88[0.82-0.94]$ & 1.00 & 0.73 & 1.00 & 0.79 \\
\hline S/F & $0.84[0.76-0.91]$ & 1.00 & 0.72 & 1.00 & 0.79 \\
\hline BG & $0.75[0.66-0.91]$ & 0.63 & 0.63 & 0.64 & 0.64
\end{tabular}

AUC, area under the curve; NPV, Positive Negative Value; PPV, Positive Predictive Value; $\mathrm{S} / \mathrm{F}, \mathrm{SpO}_{2}$ $/ \mathrm{FiO}_{2}$ ratio; $\mathrm{BG}$, blood glucose; Lym, lymphocyte counts; $\mathrm{ALT}$, alanine aminotransferase

\section{Figures}




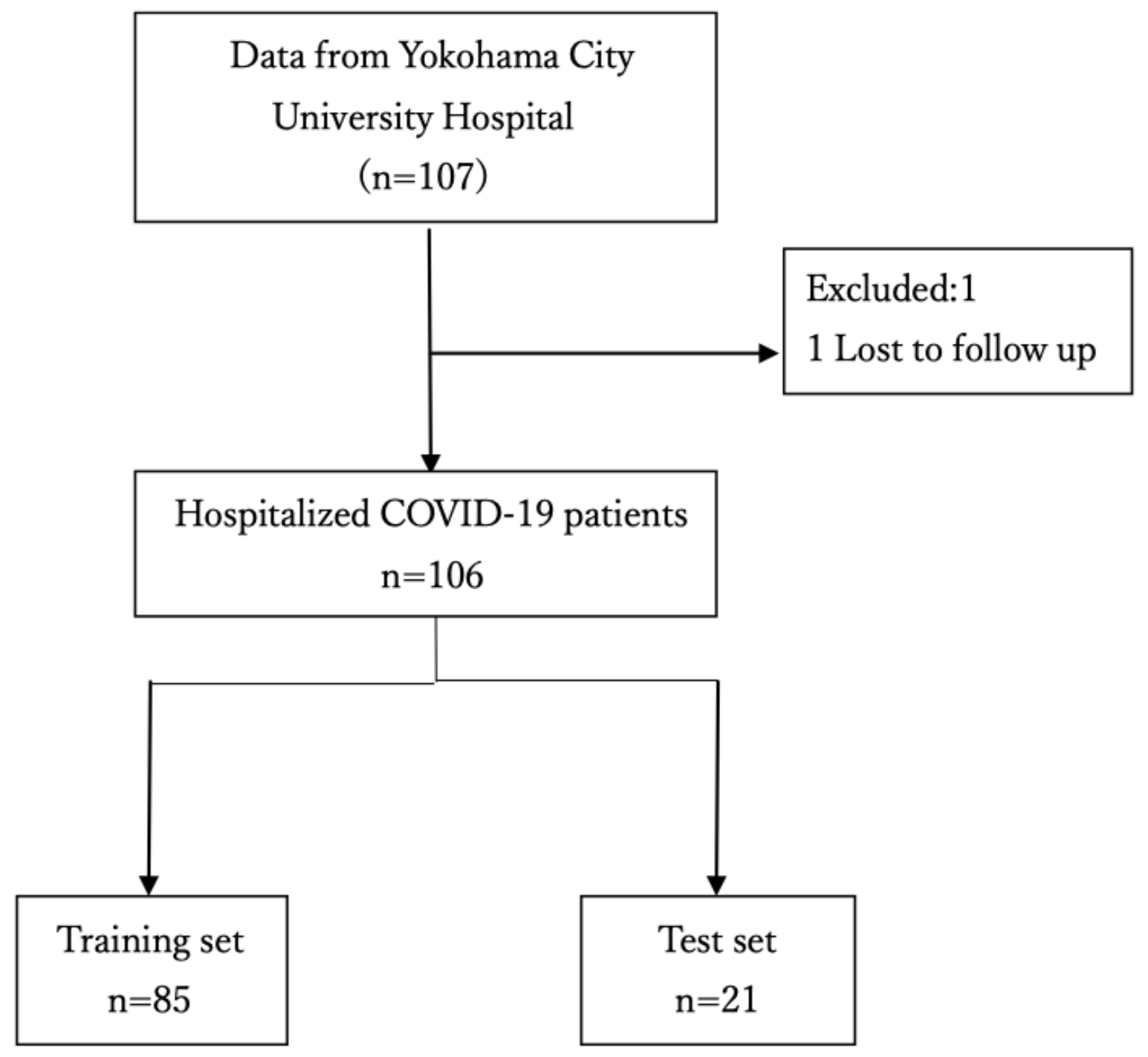

Figure 1

Patients Flow A total of 107 patients participated in the study. Finally, 106 patients were analyzed; 85 were used for training-data and 21 for test-data. 


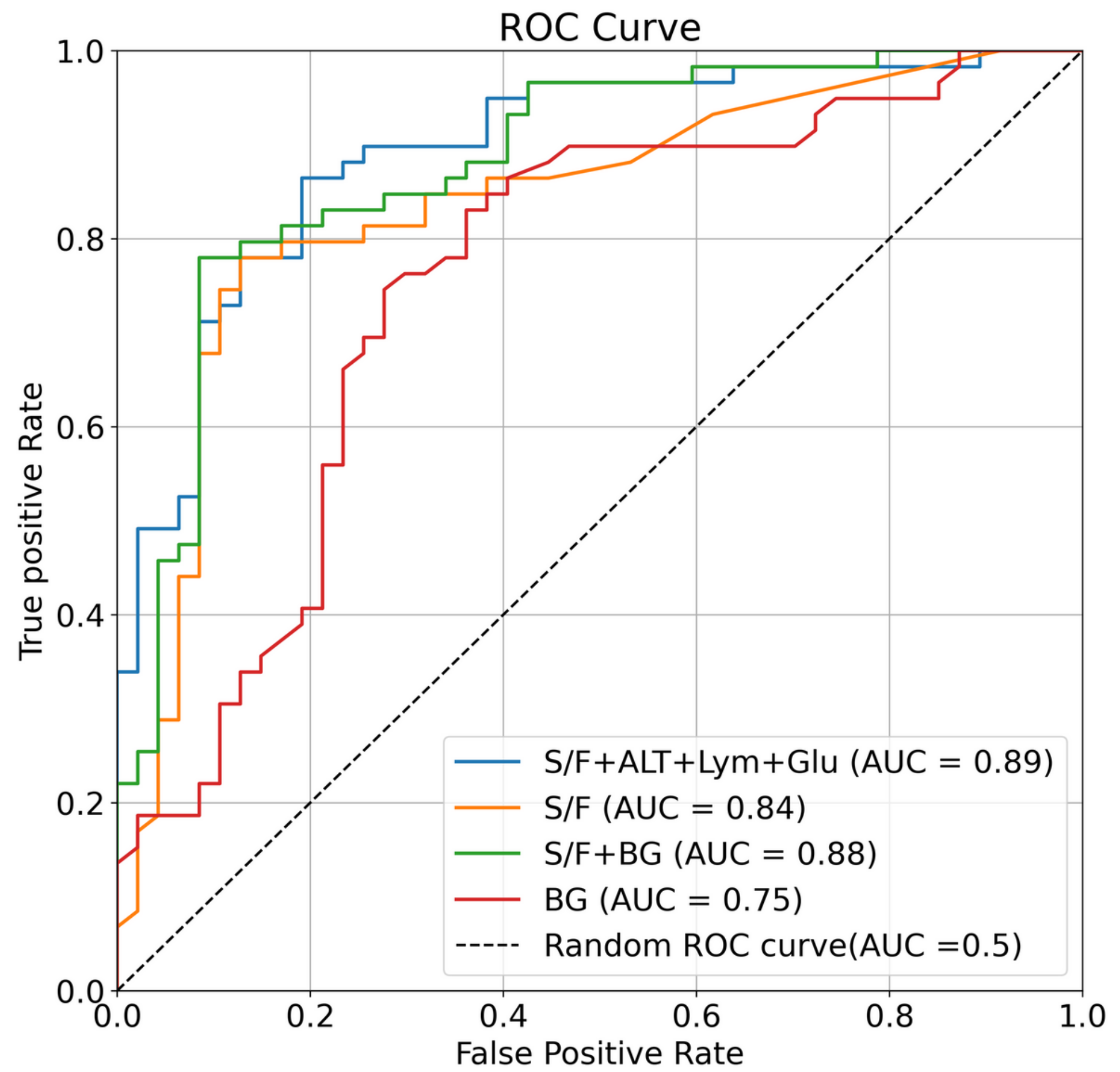

Figure 2

The Need for MV Prediction Using Simplified Logistic Regression. The area under the receiver operating characteristic curve (AUROC) of the logistic regression models (blue; S/F + Lym + ALT + BG, orange; S/F green; S/F + BG, red; BG.). S/F, SpO2 /FiO2 ratio; BG, blood glucose; Lym, lymphocyte counts; ALT, alanine aminotransferase. 


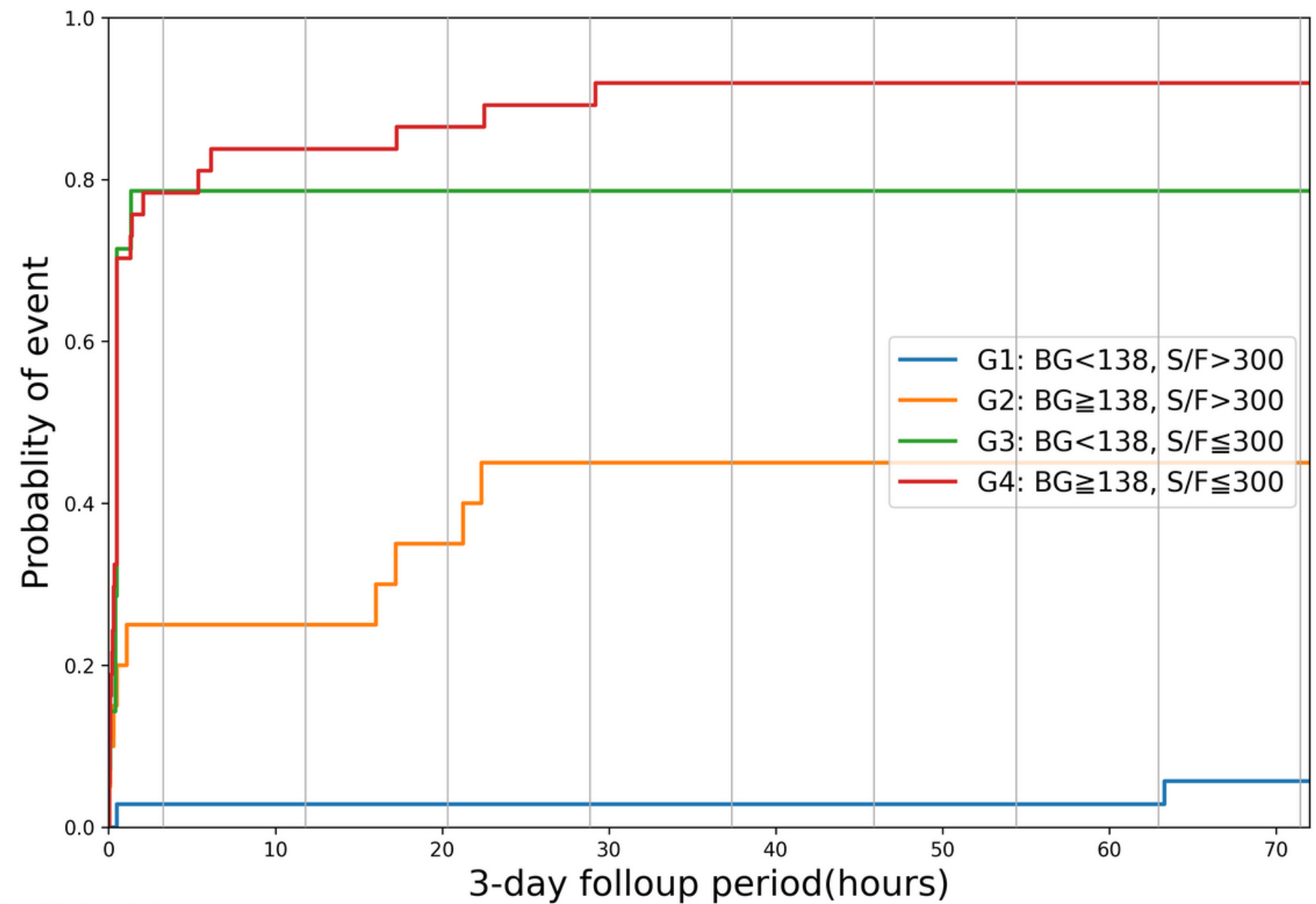

G1: $B G<138, S / F>300$

At risk 35

Censored 0

Events 0

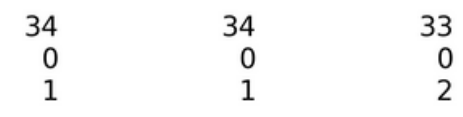

33
0
2

32
0
3

32
0
3

32
0
3

32
0
3

0
31
4

$G 2: B G \geqq 138, S / F>300$

At risk 20

Censored 0

Events 0

$\begin{array}{rr}11 & 11 \\ 0 & 0 \\ 9 & 9\end{array}$

11
0
9

11
0
9

11
0
9

11
0
9

$\begin{array}{rr}10 & 0 \\ 0 & 10 \\ 10 & 10\end{array}$

G3: $B G<138, S / F \leqq 300$

$\begin{array}{rr}\text { At risk } & 14 \\ \text { Censored } & 0\end{array}$

Events 0

3
0

3
0
11

3
0
11

3
0
11

3
0
11

3
0
11

$\begin{array}{rr}3 & 0 \\ 0 & 3 \\ 11 & 11\end{array}$

G4: $B G \geqq 138, S / F \leqq 300$

At risk 37

Censored 0

Events 0

4
0
33

3
0
34

3
0
34

3
0
34

3
0
34

3
0
34

3
0
34

0
3
34

\section{Figure 3}

Mechanical ventilation (MV) use at the 3-day follow-up period Kaplan-Meier event-incidence curves of MV use that were constructed according to above or below optimal cutoffs (blood glucose [BG]: $138 \mathrm{mg} / \mathrm{dL}$, SpO2 /FiO2 ratio [S/F ratio]: 300 ).

\section{Supplementary Files}


This is a list of supplementary files associated with this preprint. Click to download.

- supplementalfigure1.pdf 